\title{
Performance of Mathematical System Identification in Modeling of Hybrid Evaporative Cooling System
}

\author{
M.P. Islam \\ Biomechanical Systems \\ Ehime University, 3-5-7 Tarumi \\ Matsuyama 790-8566, Japan
}

\author{
T. Morimoto \\ Biomechanical Systems \\ Ehime University, 3-5-7 Tarumi \\ Matsuyama 790-8566, Japan
}

\begin{abstract}
The major aim of this study is to model the effect of outside temperature, watering, and airing on the inside temperature of a hybrid evaporative cooling system (HECS). There is great interest in evaporative cooling systems as a new storage system for fruit and vegetables for the purpose of energy saving. The heat transfer process of the HECS has not yet been well analyzed, so system identification based model was used to identify such an unknown process. Four system identification methods were used to train a three input-one output based system. After comparing the performance of the four system identification methods, continuous-time identified transfer function model was selected based on final prediction error (FPE), mean squared error (MSE), and regression of the input-output responses.
\end{abstract}

\section{General Terms}

Mathematical system identification in modeling.

\section{Keywords}

System identification, training algorithm, evaporation, passive cooling.

\section{INTRODUCTION}

An ecofriendly, comparatively new storage system called the "hybrid evaporative cooling system (HECS)", based on the principle of indirect evaporative cooling with better air circulation and water saving, has various advantages over conventional energy sources, such as short installation time and long-life operation, simplicity, no moving parts, silent, safe, non-polluting, and it is renewable source of heat and energy. Evaporative cooling remains one of the oldest, simplest, and least expensive techniques used since ancient times to delay spoilage of stored fruit and vegetables by lowering the ambient temperature. Evaporation lowers air temperature resulting in less weight loss of fresh produce, and therefore extends the shelf life of fresh agricultural produce. Dadhich et al. [1], Odesola and Onyebuchi [2], and Zahra and John [3] noted that evaporative cooling systems have potentially become attractive storage systems for fruit and vegetables.

Cooling in a HECS is mainly achieved by evaporation of water supplied to the evaporative medium between double walls. However, the cooling performance of the HECS is influenced by the combination of manipulating factors (watering and airing) and an uncontrolled factor (outside temperature). In general, water requires a large quantity of heat energy to change from a liquid to gaseous state. It is this characteristic that enables evaporating water to substantially lower the temperature of its environment. On the other hand, the amount of water vapor that can be taken up and held by the air depends on the temperature (energy level) of the air. HECS is continuously exposed to changing conditions (for example, blocking of solar radiation by clouds, underground temperature, soil moisture, deterioration of evaporative medium, thermal resistance, evaporating properties, and fruit and vegetables inside the storage area), which leads to a complex nonlinearity and a time-variation control problem requiring effective and efficient cooling. To identify the HECS heat transfer process, therefore, both the manipulating factors (watering and airing) and the uncontrolled natural factor (air temperature) were defined as the input variables and the inside temperature of the HECS as the output variable. To date, the HECS heat transfer process with a newer evaporative medium has not been well analyzed under the influence of manipulating factors and an uncontrolled natural factor.

The cooling process of a hybrid evaporative cooling system based on active and passive evaporative mechanism is nonlinear in its dynamics. Nonlinear modeling in various application areas has fewer limitations at the computational, memory, and data-acquisition levels, making nonlinear modeling a more feasible and flexible choice [4-6], and the resulting system identification is getting more attention owing to widespread development of sophisticated and efficient algorithms, coupled with the advancement of digital processing and computing [7]. System identification uses input-output relationships obtained from the experimental data of a model dynamic system.

In this study, outside temperature, watering, airing, and inside temperature are considered the basic input-output parameters for nonlinear system identification training to predict the inside temperature of a HECS. Therefore, this study included parameter identification (training), comparisons between observed and modelled HECS output (estimated inside temperature), and simulated inside temperature for predicting cooling performance.

\section{MATERIALS AND METHODS}

The cooling performance test of the experimental HECS was undertaken under no load conditions during the period from May 1, 2013 to January 31, 2015 to determine the influence of the uncontrolled natural factor (outside air temperature) and manipulating factors (airing and watering). Outside temperature, watering, airing, and their diurnal changes were investigated to identify the dynamic response of the inside temperature. An experimental hybrid evaporative cooling system (combination of active and passive evaporative mechanisms) was set up and positioned north-south, which was the average frequent air direction at the Faculty of Agriculture, Ehime University, Japan. 


\subsection{Design of the HECS}

The outside wall of the HECS (Fig. 1 a) was made of porous lava stones (PVLS; Iris Ohyama, Inc., Japan) and the inside wall was made of solid clay bricks. The storage area (Fig. 1 a) inside the HECS was $700 \mathrm{~mm}$ long $\times 700 \mathrm{~mm}$ wide $\times 500 \mathrm{~mm}$ high. The evaporating medium between the walls consisted of two layers: sand-zeolite and gravel stone (Fig. 1 a, b). The gap between the outside and inside walls was $55 \mathrm{~mm}, 40 \mathrm{~mm}$ of which was packed with filler consisting of a mixture of sand $(80 \%)$ and natural zeolite EOLITE No. 1 (ITT, Japan) (20\%), and the remaining $15 \mathrm{~mm}$ was packed with filler consisting of gravel stone $(\varnothing 2 \mathrm{~mm})$. The natural zeolite was added to the sand to increase water retention capacity and gravel stone was used to enhance the evapotranspiration rate. An air circulating tube $(\varnothing 20 \mathrm{~mm})$ was placed just beneath the outer wall, and the gravel stone and sand-zone area. A solar chimney (720 mm long $\times 800 \mathrm{~mm}$ high, Fig. 1c) made from a 3 -mm thick aluminum sheet was placed over the storage cover.

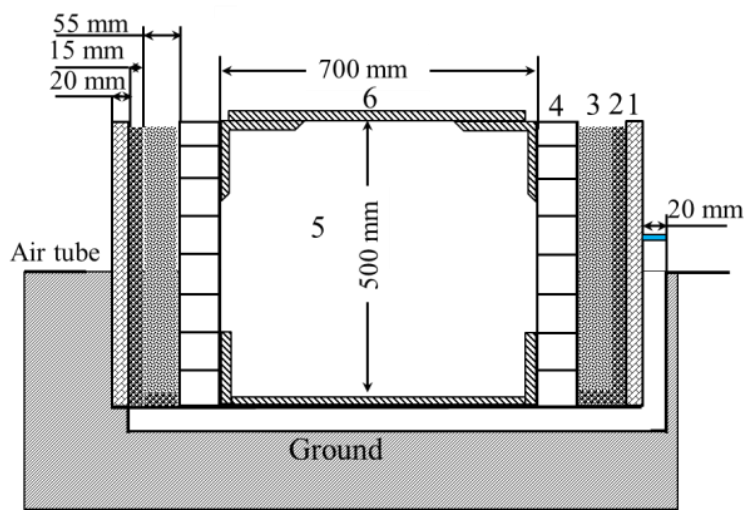

(a) Storage structure

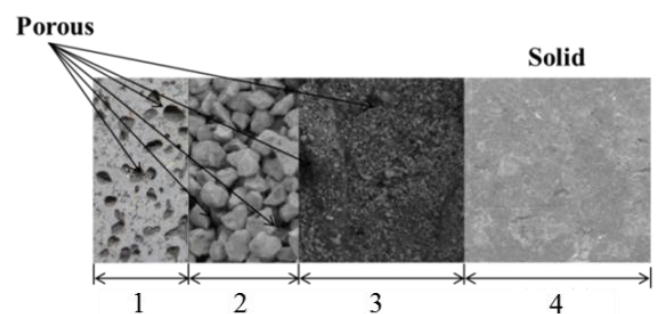

(b) Evaporative medium

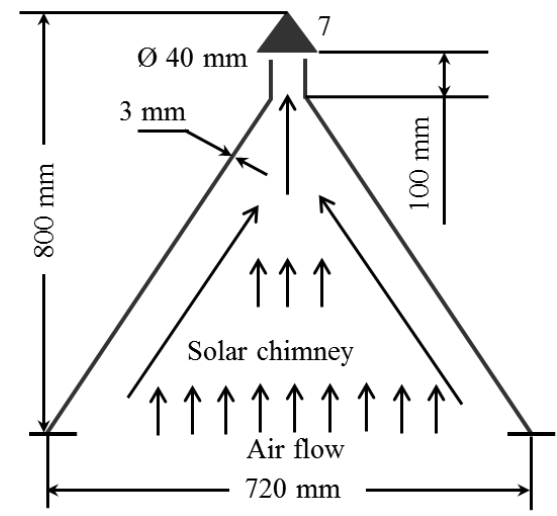

(C) Solar Chimney

(1) Outer wall, (2) Inner wall, (3) Sand-zeolite, (4) Inner wall, (5) Storage area, (6) Storage cover, (7) Exhaust vent
Tap water from an overhead $30 \mathrm{~L}$ water tank (at a height of $188 \mathrm{~mm}$ ) was supplied to the filler material through a G240 low pressure micro sprinkler (Takagi, Japan) with 0-3.7854 $\mathrm{L} \cdot \mathrm{h}^{-1}$ and a 360-degree gentle stream pattern. The amount of watering was set to $1.2 \mathrm{~L} \cdot \mathrm{h}^{-1}$. A two-port pilot kick type PKA valve (CKD Corporation, Japan) controlled by a programmable timer supplied water to the pipe. The lower and upper inside wall and bottom of the storage area was covered with a 5-mm thick polystyrene (expanded type) heat insulation board. A GFS-20I shade curtain (Takasho Cool Shade, Japan) that reduces solar radiation by $60 \%$ was also used. Outside air was circulated from the bottom to the top of the evaporation medium using a low-cost solar powered fan (Fig. 2 a). The maximum energy consumption of the fan for blowing air at $4.5 \mathrm{~m} \cdot \mathrm{s}^{-1}$ was $10 \mathrm{~W}$. The timing of the watering and airing was controlled by a programmable electronic timer.

\subsection{Working principle of the HECS}

The working mechanism of the experimental HECS (Fig. $\mathbf{2}$ a, b, c) is as follows: when the dry surface of the heat exchanging wall is in contact with air, the opposite wet surface of the same heat exchanging wall absorbs heat from the dry side and then transfers it to the evaporative medium [8]. As a result, the water molecules in the wet evaporative medium release this heat to the environment through evaporation, and therefore cool the dry side (Fig. 2 b). This method has the advantage of maintaining a lower temperature without adding further moisture to the air. Experimental observations $[9,10]$ show that under an ideal operating condition, heat flow from the product air (inside HECS) travels in a counter-flow manner to the working air (outside air) and the two airstreams have a good balance of heat flow rates over the contact area of the outside wall, evaporative medium, and inside wall of the passive evaporative cooler. As a result, the product air temperature on the dry side of the inside wall reaches the wet-bulb temperature of the incoming working air and the temperature of the working air on the wet side of the outside wall increases from its incoming dry-bulb temperature to the inside product air dry-bulb temperature and becomes saturated. Furthermore, an air circulating tube circulates outside air from bottom to top through the small porous hole in the evaporative medium. During this process, under the influence of outside unsaturated air, water molecules inside the moist evaporative medium change physical state from liquid to vapor. As a result, the air circulating tube enhances the evaporation rate of the evaporative medium.

The solar chimney (Fig. 2 c) enhances ventilation over the surface of the storage chamber. The system relies on a natural driving force, that is, solar radiation, to heat the surface of the solar chimney. The surface of the solar chimney conducts heat to the air inside the chimney, and then the heat content of the air equalizes through convection. Chakraborty and Fonseca [11] state that at constant pressure, air density decreases as temperature increases. This process continues until the air entering the solar chimney is continuously heated. Outside air passing over the surface of the wet sand-zeolite filler to enter the solar chimney enhances the evaporating process when the air absorbs more water molecules from the wet surface of the filler.

\section{Fig 1: Components of the HECS [8]}




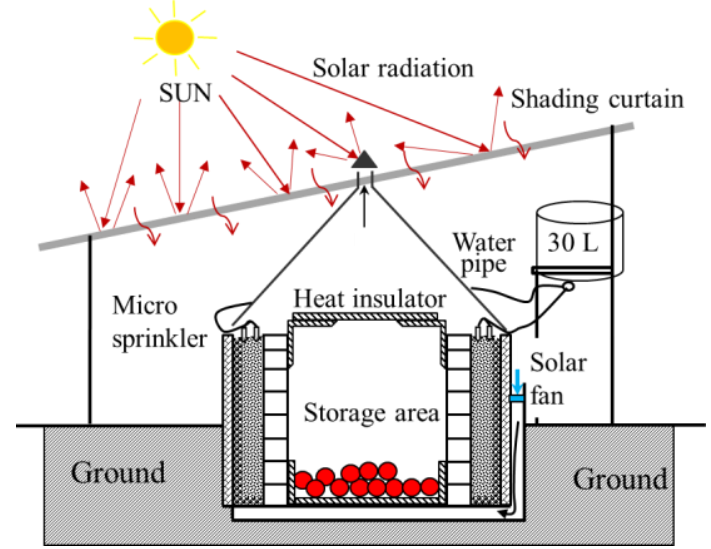

(a) Front view

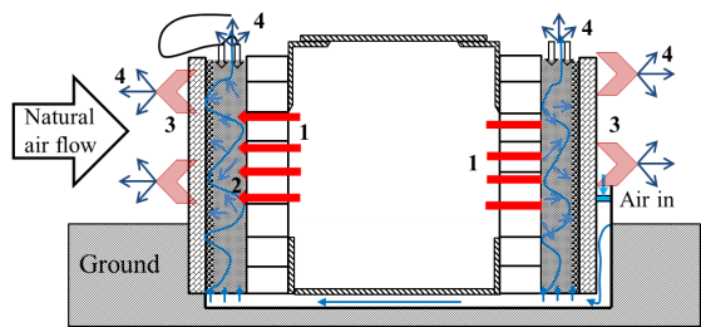

1. Heat moves out of the storage area; 2. Diffusion of water particles;

3. Latent heat of vaporization; 4. Evaporation of water

(b) View of the working mechanism

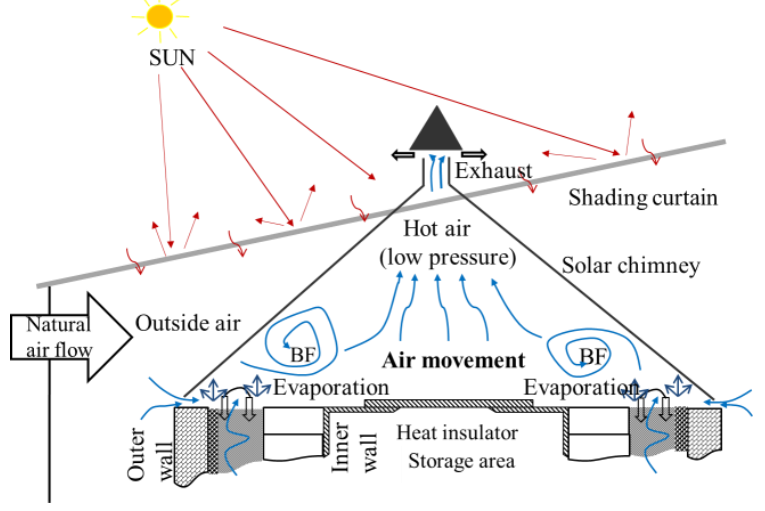

$\mathrm{BF}$ - Temperature and pressure difference between outside air and hot air inside the solar chimney creates a buoyancy force.

(c) View of the solar chimney

\section{Fig 2: Working principle of the HECS [8]}

\subsection{Modeling the HECS using a system identification method}

System identification allows the building of mathematical models of a dynamic system based on observed data. Figure 3 describes a dynamic system with object linking observable output signals, manipulatable inputs signals, and disturbances. The process of identifying a system model consists of finding mathematical functions that correlate these signals that approximate the true systematic behavior of a model. Its complexity depends on the purpose the model is designed for and is often beneficial for analysing system behaviour. The following difference equation represents a simple input-output model structure [12]:

$y(k)+a \cdot y(k-1)=b \cdot u(k)$ where $a$ and $b$ are adjustable parameters.

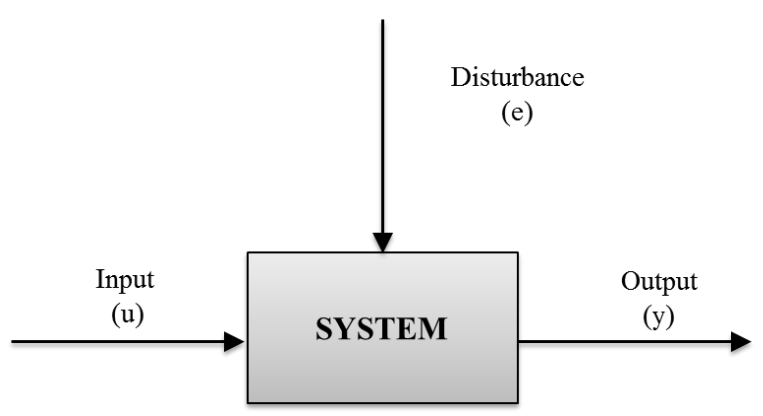

Fig 3: Dynamic model structure

The procedure for a system identification model is shown in Fig. 4. This model identification process includes the following $[13,14]$ :

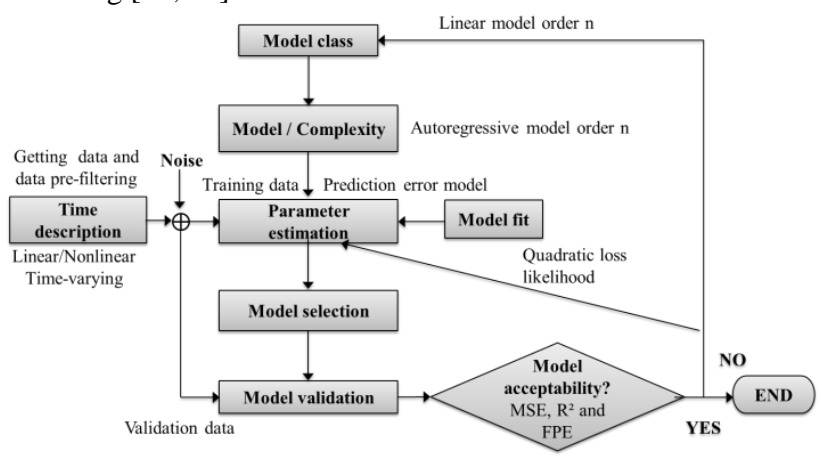

Fig 4: Flow chart for training of a system identification process

\subsubsection{Training algorithms}

The following methods were used in this study:

\subsubsection{Continuous-time identified transfer function model (TF)}

In continuous time, a transfer function model has the following form [12]:

$y(s)=\frac{\operatorname{num}(s)}{\operatorname{den}(s)} \cdot u(s)+e(s)$

where $y(s), u(s)$, and $e(s)$ represent the Laplace transforms of the output, input, and noise, respectively. Parameters num(s) and $\operatorname{den}(s)$ represent the numerator and denominator polynomials that define the relationship between the input and the output.

\subsubsection{Process model (PD)}

The structure of a process model is a simple continuous-time transfer function that describes linear system dynamics. The following model structure is a first-order continuous-time process model [12]:

$G(s)=\frac{K_{p}}{1+s \cdot T_{p 1}} \cdot e^{-s \cdot T_{d}}$

where $K_{p}$ is the static gain, $T_{p 1}$ is a time constant, and $T_{d}$ is the input-to-output delay.

\subsubsection{Polynomial model (BJ)}

A polynomial model uses a generalized notion of transfer functions to express the relationship between the input $u(t)$, the output $\mathrm{y}(\mathrm{t})$, and the noise $\mathrm{e}(\mathrm{t})$ using the following equation [12]: 
$A(q) \cdot y(t)=\sum_{i=1}^{n u} \frac{B_{i} \cdot(q)}{F_{i} \cdot(q)} \cdot u_{i}\left(t-n \cdot k_{i}\right)+\frac{C(q)}{D(q)} \cdot e(t)(4)$

where the variables $A, B, C, D$ and $F$ are polynomials expressed in the time-shift operator $\mathrm{q}^{-1}$. Parameter $u_{i}$ is the $i$ th input, $n u$ is the total number of inputs, and $n \cdot k_{i}$ is the ith input delay that characterizes the transport delay. The variance of the noise $e(t)$ is assumed to be $\lambda$.

\subsubsection{Correlation model (IMP)}

Impulse response is the output signal that results when the input is an impulse and has the following definition for a discrete model [12]:

$$
\begin{array}{ll}
u(t)=0 & t>0 \\
u(t)=1 & t=0
\end{array}
$$

The response to an input $u(t)$ is equal to the convolution of the impulse response, as follows:

$y(t)=\int_{0}^{t} h(t-z) \cdot u(z) d z$

\subsection{Measurements}

Temperature was measured using two 47SD digital thermometers with data logger function (Sato Shoji Inc., Japan), each with four thermocouples ( $00.3 \mathrm{~mm}$ ) having an accuracy of $\pm 0.1^{\circ} \mathrm{C}$. Solar radiation was measured using a solar power meter with data logger function (SPM-SD; Sato Shoji Inc., Japan). Air speed was measured using two AM14SD digital anemometers with data logger (Sato Shoji Inc., Japan). A Revex PT60D programmable timer (Ningbo Bainian Electric Appliance Co. Ltd., China) was used to control watering during the experiment period. These data were recorded at intervals of $60 \mathrm{~s}$ for $24 \mathrm{~h}$ resulting in 1440 readings per day. The system identification methods were examined and compared using MATLAB ${ }^{\circ}$ environment (version R2014a, The MathWorks Inc., Natick, MA, USA), and System Identification Toolbox.

\section{RESULTS AND DISCUSSION}

\subsection{Diurnal changes in the inside} temperature of the HECS for identification

First, a data-set consisting of 4200 data points for the input and output variables was obtained for identification from a real system. Figure 5 shows typical diurnal changes in the outside temperature, watering, airing, and inside temperature of the HECS. From the figure, it can be seen that under the combination of on-off watering and airing and only airing, the average inside temperature was $20.7^{\circ} \mathrm{C}$ and $28.7^{\circ} \mathrm{C}$, respectively. Under watering and airing, evaporated water molecules separate at a faster rate from the wet evaporative medium, resulting in a further decrease in inside temperature of the HECS. This result shows that watering and airing enhances the evaporation process. Therefore, we chose watering and airing as the input variables for system identification.

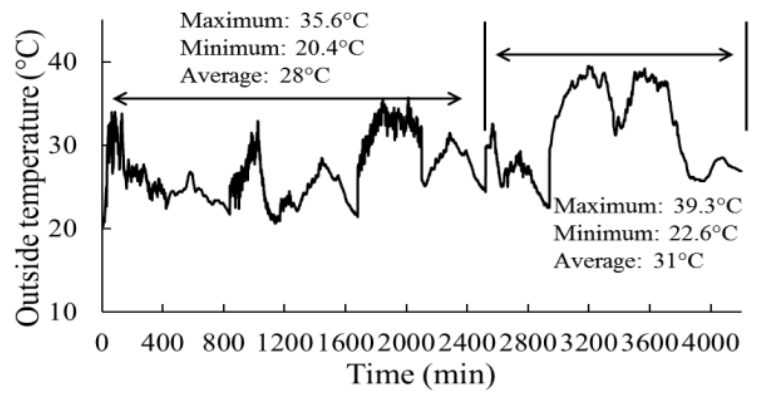

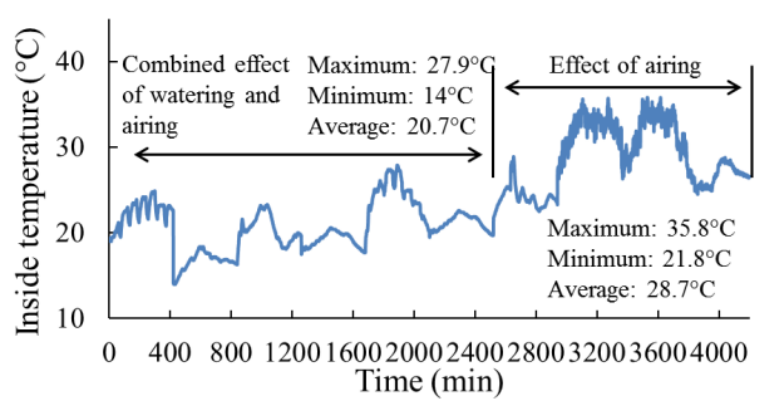

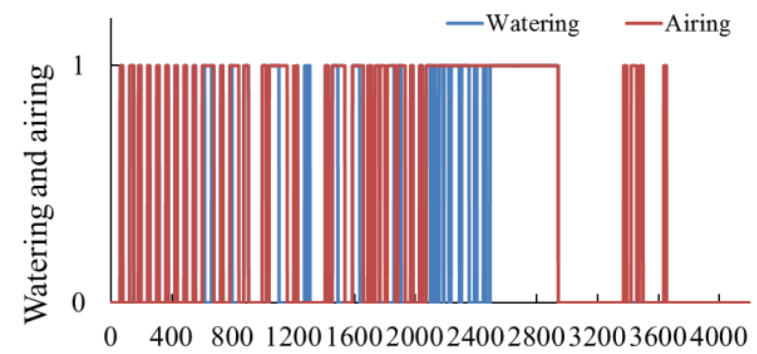
Time ( $\mathrm{min})$

Fig 5: Diurnal changes in outside temperature and inside temperature in the HECS under watering and airing conditions

\subsection{Determination of training performance in system identification system}

Figure 6 shows the best resulting system identification network model based on the average performance of final prediction error (FPE), mean squared error (MSE), and regression of the input-output responses. The graph shows that the large values for regression (72.99), lower value of MSE (0.67), and lower value of FPE (1.63) obtained using the continuous-time identified transfer function model (TF).

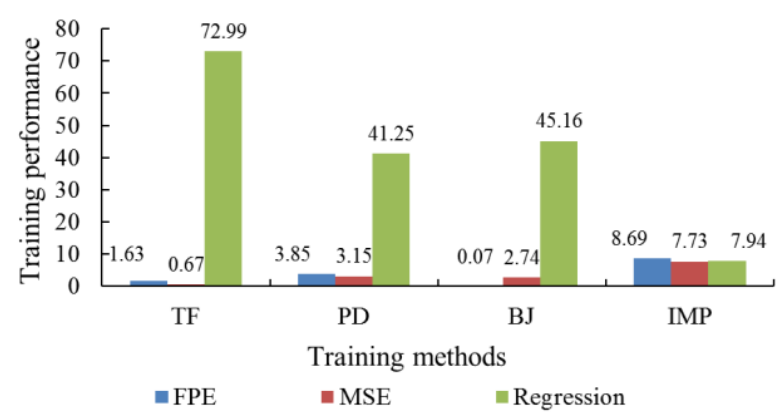

Fig 6: Training performance in the system identification system

\subsection{The simulated observed and estimated model output of the continuous-time identified transfer function model}

Figures 7 a, b show the comparison between the observed and estimated relationships of the inside temperature. The estimated relationship was obtained from system identification model simulation using the continuous-time identified transfer function. All the data in this graph were obtained from the calculation of the step responses of the inside temperature as affected by various levels of the step input of the outside temperature, watering, and airing. It can be seen that the estimated-observed relationship obtained from the continuous-time identified transfer function $\left(\mathrm{R}^{2}=0.91\right)$ was closely related to the observed relationship. In 
general, it is well known that the relationship between input and output shows nonlinear characteristics because of the thermo-physical properties of the evaporating mechanism of the HECS and environmental factors. These results suggest that a reliable computational model could be obtained for predicting the behavior of the inside temperature using the continuous-time identified transfer function model under any combination of outside temperature, watering, and airing.

\subsection{Comparison of the dynamic and static observed and predicted value of the} continuous-time identified transfer function

The observed and estimated predicted values between observed inside temperature and predicted inside temperature are shown in Fig. 8 a, b. These values are obtained from the stationery values of step responses of net inside temperature as affected by the various gains in step input of outside temperature, watering, and airing by using the nonlinear dynamic model method. It can be seen that the estimated relationship was closely related to the observed relationship (dynamic relationship: $\mathrm{R}^{2}=0.9421$ and static relationship: $\mathrm{R}^{2}=0.9527$ ). The nonlinear characteristics appear in both curves. This result implies that the nonlinear identification of the inside temperature was well attained using the continuoustime identified transfer function.

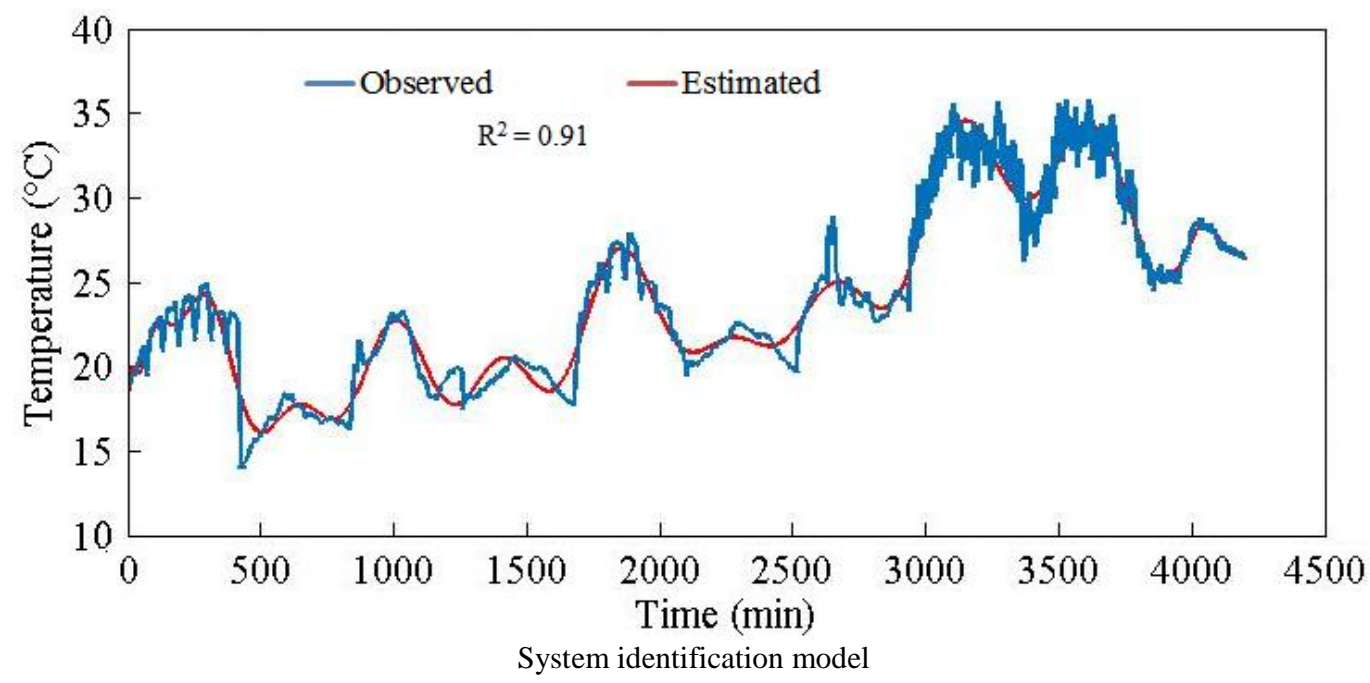

Fig 7: Dynamic nonlinear relationship between observed and estimated results by simulation

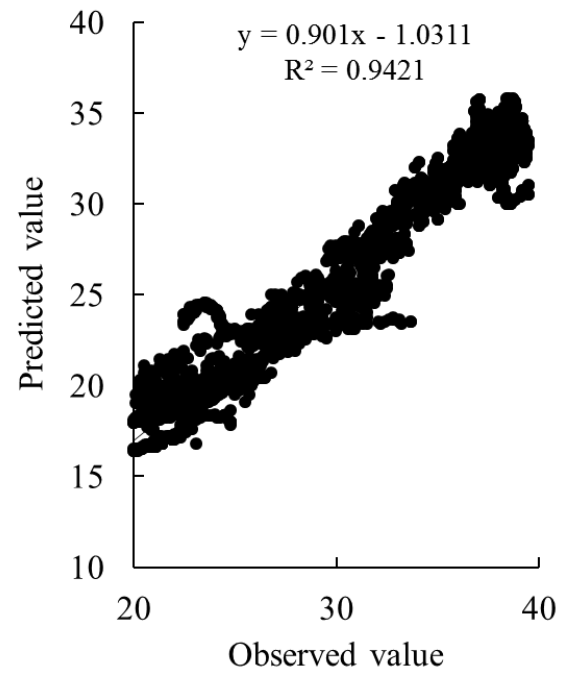

(a) Dynamic relationship

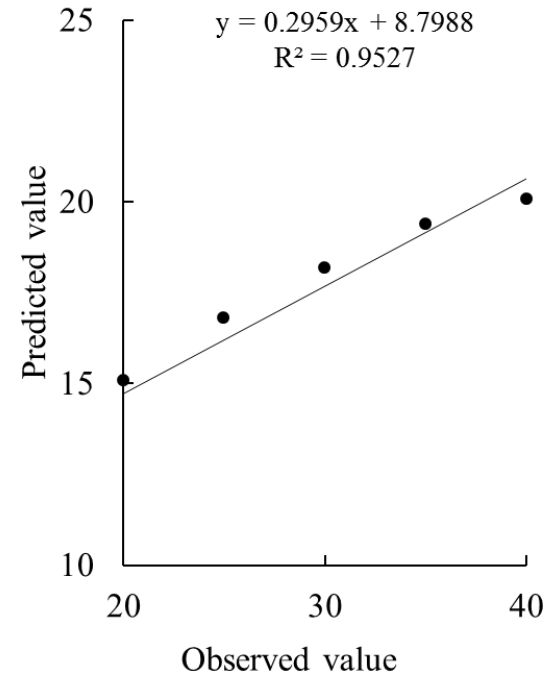

(b) Static relationship

Fig 8: Nonlinear relationships between observed and estimated values

\section{CONCLUSION}

A hybrid evaporative cooling system (HECS) consists of double walls made of lava plates and bricks, an evaporative medium (gravel stone-sand-zeolite) between the double walls, a half below ground storage space, a water supply, and an airing system from bottom to top through the evaporative medium. The HECS works on the direct and indirect evaporative cooling principle. Cooling of the HECS is achieved by applying water to the evaporative medium and airing. On-off watering and airing reduced the inside temperature of the HECS. Four training methods for system identification consisting of the continuous-time identified transfer function model, process model, polynomial models, and correlation model have been evaluated. First, the precision of confidence performance based on regression 
value was measured by simulating observed and estimated model output of the continuous-time identified transfer function model and we found that the simulated estimated relationship was closely related $\left(\mathrm{R}^{2}=0.91\right)$ to the observed relationship trained by the continuous-time identified transfer function. Later, the precision of predictive ability was measured and we found that the static and dynamic relationships between observed and estimated values were close to the real relationship. Finally, the results of this study indicate that the continuous-time identified transfer function model can be used as the best training algorithm for predicting the cooling performance of a HECS by reducing time in testing of prototypes in real time.

\section{ACKNOWLEDGMENTS}

We would like to acknowledge the financial support provided by the Basic Science Research Projects from the Sumitomo Foundation (No. 143039).

\section{REFERENCES}

[1] Dadhich S.M., Dadhich H. and Verma R.C. 2008. Comparative study on storage of fruits and vegetables in evaporative cool chamber and in ambient. Int $\mathrm{J}$ Food Eng., 4(1): 1-11. DOI: 10.2202/1556-3758.1147

[2] Odesola I.F. and Onyebuchi O. 2009. A review of porous evaporative cooling for the preservation of fruits and vegetables. Pacific J Sci Technol., 10(2): 935-941.

[3] Zahra G. and John A.B. 1996. A passive evaporative cooling system by natural ventilation. Build Environ, 31(6): 503-507.

[4] Soni A.S. 2006. Control-relevant system identification using nonlinear volterra and volterra-laguerre models. $\mathrm{PhD}$ Thesis, Graduate Faculty of the School of Engineering, University of Pittsburgh.

[5] Paduart J., Lauwers L., Swevers J., Smolders K., Schoukens J. and Pintelon R. 2010. Identification of nonlinear systems using polynomial nonlinear state space models. Automatica, 46(4): 647-656. DOI: 10.1016/j.automatica.2010.01.001

[6] Paduart J. 2008. Identification of nonlinear systems using polynomial nonlinear state space models. PhD Thesis, Vrije Universiteit, Brussels.

[7] Shariff H.M., Rahiman M.H.F., Yassin I. and Tajjudin M. 2014. System identification of a steam distillation pilot-scale using arx and narx approaches. IJRET: International Journal of Research in Engineering and Technology, 3(1): 144-157.

[8] Islam M.P. and Morimoto T. 2015. Evaluation of a new heat transfer and evaporative design for a zero energy storage structure. Solar Energy, 118: 469-484.

[9] Stoitchkov N.J. and Dimitrov G.I. 1998. Effectiveness of cross flow plate heat exchanger for indirect evaporative cooling. Int J Refrig., 21(6): 463-471.

[10] Liu X., Li Y., Li J., Yang H. and Chen H. 2009. Efficiency analysis of cross-flow plate heat exchanger for indirect evaporative cooling sustainability. Sustainability in Energy and Buildings, 255-264. DOI:10.1007/978-3642-03454-1_26

[11] Chakraborty J. and Fonseca E. 2005. Analysis and evaluation of a passive evaporative cool tower in conjunction with a solar chimney. In the proceedings of the 22nd PLEA (Passive and Low Energy Architecture) nternational Conference, 1-6, 13-16 November, Notre Dame University, Beirut, Lebanon.

[12] Ljung L. 2014. System Identification Toolbox ${ }^{\mathrm{TM}}$ Getting Started Guide. MATLAB \& SIMULINK®. The MathWorks, Inc.

[13] Soderstrom T. and Stoica P. 1989. System Identification, Prentice-Hall.

[14] Thakur A., Sankhe A., Prajapati R., Saha A. and Salvi M. 2013. System identification. International Journal of Students Research in Technology \& Management, 1(3): 270-277. 